\title{
Comparative genome-wide polymorphic microsatellite markers in Antarctic penguins through next generation sequencing
}

\author{
Juliana A. Vianna ${ }^{1,2}$, Daly Noll ${ }^{1}$, Isidora Mura-Jornet ${ }^{1}$, Paulina Valenzuela-Guerra ${ }^{1}$, \\ Daniel González-Acuña ${ }^{3}$, Cristell Navarro ${ }^{4}$, David E. Loyola ${ }^{4}$ and Gisele P.M. Dantas ${ }^{5}$ \\ ${ }^{1}$ Departamento de Ecosistemas y Medio Ambiente, Pontificia Universidad Católica de Chile, Santiago, \\ Chile. \\ ${ }^{2}$ Centro de Cambio Global UC, Santiago, Chile. \\ ${ }^{3}$ Departamento de Ciencias Pecuarias, Facultad de Ciencias Veterinarias, Universidad de Concepción, \\ Chillán, Chile. \\ ${ }^{4}$ Centro Nacional de Genómica y Bioinformática, Santiago, Chile. \\ ${ }^{5}$ Pontificia Universidade Católica de Minas Gerais, Belo Horizonte, MG, Brazil.
}

\begin{abstract}
Microsatellites are valuable molecular markers for evolutionary and ecological studies. Next generation sequencing is responsible for the increasing number of microsatellites for non-model species. Penguins of the Pygoscelis genus are comprised of three species: Adélie ( $P$. adeliae), Chinstrap ( $P$. antarcticus) and Gentoo penguin ( $P$. papua), all distributed around Antarctica and the sub-Antarctic. The species have been affected differently by climate change, and the use of microsatellite markers will be crucial to monitor population dynamics. We characterized a large set of genome-wide microsatellites and evaluated polymorphisms in all three species. SOLiD reads were generated from the libraries of each species, identifying a large amount of microsatellite loci: 33,677, 35,265 and 42,057 for $P$. adeliae, $P$. antarcticus and $P$. papua, respectively. A large number of dinucleotide $(66,139)$, trinucleotide $(29,490)$ and tetranucleotide $(11,849)$ microsatellites are described. Microsatellite abundance, diversity and orthology were characterized in penguin genomes. We evaluated polymorphisms in 170 tetranucleotide loci, obtaining 34 polymorphic loci in at least one species and 15 polymorphic loci in all three species, which allow to perform comparative studies. Polymorphic markers presented here enable a number of ecological, population, individual identification, parentage and evolutionary studies of Pygoscelis, with potential use in other penguin species.
\end{abstract}

Keywords: microsatellite markers, penguin, genome, polymorphism, tetranucleotide.

Received: August 22, 2016; Accepted: March 17, 2017.

\section{Introduction}

The high level of polymorphism in microsatellites, or short tandem repeats (STRs), coupled with their codominant nature, is advantageous for population genetics or behavioral studies, such as paternity tests, investigation of mating patterns, phylogeography, etc. However, the identification of microsatellites in non-model species by traditional methods is a time-consuming process requiring extensive laboratory procedures and Sanger sequencing to achieve isolation of only a few loci per surveyed genome. The restricted number of loci identified through traditional methods may limit the results of a study. Therefore, most investigations on non-model organisms rely on cross-am-

Send correspondence to Juliana A. Vianna. Departamento de Ecosistemas y Medio Ambiente, Facultad de Agronomía e Ingeniería Forestal, Pontificia Universidad Católica de Chile. Av. Vicuña Mackenna 4860, Santiago, Chile. E-mail: jvianna@uc.cl. plification of microsatellite loci from closely related species, a procedure whose applicability decreases as the evolutionary divergence among taxa increases. This is the case of bird species, for which microsatellite loci are much less abundant than for other vertebrate classes (Primmer et al., 1997; Neff and Gross, 2001). Dawson et al. (2010) evaluated microsatellites across several bird species belonging to 15 orders and selected cross-species markers, thus reducing the costs and time associated with developing new ones. However, the use of microsatellites developed for different species is subject to scoring errors, such as null alleles, which can lead to biases in ecological and evolutionary conclusions drawn from the data (Dewoody et al., 2006). Null alleles occur when one allele does not amplify because of mutations in the sequence where a primer was designed to anneal, which can often occur with unspecific primers (Dakin and Avise, 2004). On the other hand, using different microsatellites developed de novo for each species limits 
prospects of comparative studies between taxa. For that reason, identification of microsatellite loci common for a few closely related species of interest, allows comparative ecological and evolutionary inquiries.

Recent Next Generation Sequencing (NGS) technologies make it feasible to obtain a large number of markers (e.g. microsatellites, SNPs) and have thus revolutionized molecular studies in non-model organisms, permitting rapid characterization of gene structure and expression (Ellegren, 2008). Studies using neutral microsatellites can provide information to understand aspects of species' ecology and population genetic structure (Freer et al., 2015; Vianna et al., 2017). Moreover, behavioral differences between males and females are frequently interpreted comparing population genetic patterns obtained from different markers such as mtDNA (maternal lineage) and microsatellite loci (biparental lineage; Freer et al., 2015; Vianna et al., 2017). An increasing number of genomes have recently become available, including those of several bird species (Jarvis et al., 2014; Zhang et al., 2014), with two species of penguins among them (Li et al., 2014). Those studies focus on genome description and structure, phylogeny, adaptation, and comparative analyses. Although a large number of microsatellite loci are becoming available for new genomes, they are seldom evaluated with regard to their level of polymorphism in related species.

Antarctic organisms have been strongly affected by climate change during the past 50 years, mostly in the West Antarctic Peninsula (WAP), where effects are more drastic (e.g. Croxall et al., 2002; Meredith and King, 2005; Montes-Hugo et al., 2009; Trathan et al., 2011). Genomic studies using NGS technology can, thus, provide different molecular markers to help understand adaptation, population dynamics and behavior of Antarctic taxa.

Penguins represent a monophyletic group from the Spheniscidae family, encompassing 18 species distributed exclusively in the Southern Hemisphere (Stonehouse, 1975; Williams, 1995). Pygoscelis penguins are comprised of three species, the Adélie penguin ( $P$. adeliae), the Chinstrap penguin ( $P$. antarcticus) and the Gentoo penguin $(P$. papua), all adapted to the cold temperatures around Antarctica and the sub-Antarctic islands. Among the three living species, $P$. papua has the most northern distribution along the Antarctic Peninsula and sub-Antarctic islands. $P$. antarcticus has a more southern distribution almost exclusively around the Antarctic Peninsula, and P. adeliae is the most dependent on ice, reaching higher latitudes and a circumpolar distribution (Stonehouse, 1975). P. adeliae, $P$. papua and $P$. antarcticus are classified by the IUCN as Least Concern (IUCN - International Union for Conservation of Nature, 2017).

Molecular DNA studies of Pygoscelis were mostly restricted to sequences (Ritchie and Lambert, 2000; Ritchie et al., 2004; Peña et al., 2014; Clucas et al., 2014), except for a study of $P$. adeliae using six dinucleotide micro- satellites (Roeder et al., 2001). These were developed by traditional methods using genomic libraries (Roeder et al., $2001 ; 2002)$ and then investigated for cross-amplification in 16 other species of penguins, most of which were monomorphic (Roeder et al., 2002). Recently, Kang et al. (2015) isolated sixteen polymorphic microsatellite loci for the chinstrap penguin (P. antarcticus), while Li et al. (2014) sequenced the genomes of $P$. adeliae and Aptenodytes forsteri to study the phylogenetic and population history of penguins, but neither evaluated microsatellites for polymorphisms.

The three Pygoscelis species in this study have suffered the effects of climate change, with reductions in population size, changes in distribution, and even local extinction. The WAP, a large area where these species are found, is the Antarctic region that has been most affected by climate change. Therefore, genetic studies of population structure, using variable molecular markers, are important in monitoring these Antarctic species in space and time.

We sequenced the genomes of Pygoscelis adeliae, $P$. antarcticus and P. papua using the Applied Biosystems Support Oligonucleotide Ligation Detection (SOLiD) platform, and identified and characterized microsatellite loci evaluating and comparing structure (motif class and type) among species. We also report a genome-wide set of several microsatellite loci for cross amplification in all three Pygoscelis species and the evaluation of the degree of polymorphism of several tetranucleotide loci. Finally, we report a set of primers designed for several microsatellite loci. These have similar melting temperatures and allow multiplex amplification using the same PCR protocol. These loci can be an important resource for future genetic studies of penguin populations to help management and conservation of these species in the face of climatic change.

\section{Material and Methods}

\section{SOLiD sequencing}

Genomic DNA was isolated from Pygoscelis papua, $P$. antarcticus and $P$. adeliae blood samples preserved in ethanol using the salt method (Aljanabi and Martinez 1997). DNA from six individuals belonging to the three species was quantified and quality checked by fluorometry using the PicoGreen®assay kit (Invitrogen). Genomic sequencing in ABISOLiD $5500 \mathrm{XL}$ was performed at Omics Solution, a Next Generation Sequencing facility (Santiago, Chile). DNA was desalted and then concentrated using standard EtOH/sodium acetate precipitation at $20^{\circ} \mathrm{C}$ for 2 $\mathrm{h}$, followed by two $70 \% \mathrm{EtOH}$ washes. The pooled DNA was re-dissolved in low TE as per standard protocol for ABI SOLiD sequencing of genomic DNA fragment libraries. DNA samples were sheared in a CovarisS220 System (Thermo Fisher Scientific), which sonicates the input DNA into small fragments with a mean size of around $160 \mathrm{bp}$. 
The fragmented DNA was then purified with SOLiD Library Column Purification Kit (Thermo Fisher Scientific), and libraries were prepared according to standard SOLiD protocols. Fragment libraries for the twelve penguin samples were prepared separately as follows: P1 and P2 adapters (Thermo Fisher Scientific) were ligated, and each sample was tagged with a different barcode (a known adapters sequence of ten bp). Prepared libraries were quantified by real-time PCR in a Light Cycler ${ }^{\circledR N a n o}$ (Roche) using the Quantification Kit for SOLiD (Invitrogen). Each double stranded library was added at a concentration of 0.2 $\mathrm{pg} / \mu \mathrm{L}$ to the emulsion with 2,400 million beads, according to the manufacturers' instructions. Thirty percent of the beads were P2 positive (contained amplified library fragments) before enrichment, and $90 \%$ of the beads were $\mathrm{P} 2$ positive after enrichment, yielding 790 million beads deposited in the Flow Chip. Library beads were sequenced in a SOLiD $5500 \mathrm{XL}$ using standard chemistry for paired-end fragment libraries and 35-75 bp read lengths.

\section{SOLiD sequence alignment}

The color-space reads (di-base encoded) were aligned with LifeScope software (Applied Biosystems) using the genome assembly of $P$. adeliae (Li et al., 2014) as reference. The reference was translated into color-space with the aim of mapping the reads. The color-space reads helped to improve the quality of each base call, since each base was read twice during the sequencing step.

The consensus sequence was built from the binary alignment map (BAM) files obtained in the previous step. We used the SAMtools (Li et al., 2009) repositories to obtain all bases mapped to each position, BCF tools to get the most probable genotype per position, and VCF utilities to build the consensus sequence in FASTQ format. The FASTQ file was then converted to FASTA using SEQTK (Li, 2012).

\section{Identification of microsatellite loci}

The search for dinucleotide, trinucleotide, and tetranucleotide tandem repeats in the obtained scaffolds were done using MISA software (http://pgrc.ipkgatersleben.de/misa/). We designed primers to amplify repeat fragments of $200 \mathrm{bp}$ or less. Oligonucleotide primer pairs flanking the microsatellite sequence were designed using Primer 3.0 software (Rozen and Skaletsky, 2000; http://primer3.sourceforge.net/) based on the following parameters: product size of $150-250$ and $250-300 \mathrm{bp}$; TM of $60{ }^{\circ} \mathrm{C}$, ranging between 58 and $63^{\circ} \mathrm{C}$. Reverse-complement repeat motifs (e.g. TG and $\mathrm{CA})$ and translated or shifted motifs (e.g. TGG and GTG) were grouped together such that there were a total of four, 10 and 33 unique dinucleotide, trinucleotide and tetranucleotide repeats, respectively.

\section{Microsatellite isolation and evaluation}

From our list of potential primers present in P. papua (Gentoo penguin, GP), P. antarticus (Chinstrap penguin, $\mathrm{CP}$ ) and $P$. adeliae (Adélie penguin, AP) (Table S1), we chose a subset for evaluation in all three species. The first criterion for selection was a tetranucleotide, simple and perfect SSR repeat motif and TM of $60^{\circ} \mathrm{C}$. Primer pairs for each locus were evaluated using the AmplifX v. 1.5.4 software (http://crn2m.univ-mrs.fr/pub/amplifx-dist) considering the stability of the PCR reaction, percentage of GC, stability at 3', the absence of dimers or hairpins. Although tetranucleotide microsatellites may have lower mutation rates than dinucleotides (Kruglyak et al., 1998; Schug et al., 1998), we decided to carry on our analysis using polymorphic tetranucleotides that amplify in all three penguin species. Amplification of artifact bands (or stutter bands) in dinucleotide repeats can lead to misidentification of an allele, something that is less likely to happen in tetranucleotide repeats. Tetranucleotide microsatellites have shown higher discriminatory power among closely related populations than most dinucleotides (e.g., Greig et al., 2003; Huang et al., 2015). Hence, tetranucleotides are becoming increasingly popular markers because allele differences are easier to distinguish than those of dinucleotide repeats.

Therefore, 170 loci of tetranucleotide SSR repeat motifs were selected to evaluate polymorphisms in each Pygoscelis species. PCR reactions incorporated the forward primers with 5'-end-M13 tail, the fluorophorelabeled M13 primer (Schuelke, 2000) with 6-FAM, NED or HEX (Applied Biosystems) and a reverse primer. The analysis was done on 26 samples, nine from $P$. papua, nine from $P$. antarcticus and eight from $P$. adeliae, with individuals from three, three and two different sampling locations, respectively.

We searched for orthology for all tetranucleotide loci between pairwise species and all three species considering $100 \%$ identity between the primers sequences using the option Find Duplicates in Excel and the criteria of location at the same scaffold and same locus motif.

Polymerase chain reactions (PCRs) were carried out in a $30 \mu \mathrm{L}$ volume containing $2 \mu \mathrm{L}$ of DNA at $25 \mathrm{ng} / \mu \mathrm{L}, 1 \mathrm{X}$ reaction buffer, $1.5 \mathrm{mM}$ of $\mathrm{MgCl}_{2}, 200 \mathrm{uM}$ of each dNTP, $0.4 \mu \mathrm{M}$ of each primer, and 0.8 units of Taq DNA polymerase (Brasil, Invitrogen). The PCR protocol was as follows: 10 min at $95{ }^{\circ} \mathrm{C}$, a touchdown series of $95{ }^{\circ} \mathrm{C}$ for $15 \mathrm{~s}$, $60-50{ }^{\circ} \mathrm{C}$ for $30 \mathrm{~s}, 72{ }^{\circ} \mathrm{C}$ for $45 \mathrm{~s}$, with two cycles at each annealing temperature, and 35 amplification cycles of 95 ${ }^{\circ} \mathrm{C}$ for $15 \mathrm{~s}, 50{ }^{\circ} \mathrm{C}$ for $30 \mathrm{~s}, 72^{\circ} \mathrm{C}$ for $45 \mathrm{~s}$, followed by a final extension step of 30 min at $72{ }^{\circ} \mathrm{C}$. All PCR products were loaded on 3\% agarose gels with SB buffer (Brody and Kern, 2004), and also on non-denaturing $12 \%$ acrylamide gels (99:1 acrylamide: bis-acrylamide), and run for $0.5 \mathrm{~h}$ at $300 \mathrm{~V}$, and $3 \mathrm{~h}$ at $300 \mathrm{~V}$, respectively. Bands in agarose gels 
were visualized with GelRed on an UV transilluminator, and acrylamide gels were stained with silver nitrate.

For our purposes, PCR amplification of a microsatellite was considered successful if a band of the expected size was observed after gel electrophoresis, even if two or more bands were amplified. Monomorphic tetranucleotide loci observed were discarded, and all loci with more than one allele were genotyped at Macrogen Inc. (Korea). Electrophoretograms were analyzed using GeneMarkerv1.75 (Softgenetics LLCTM, State College). A microsatellite locus was considered successful if the resulting electrophoretograms showed at least two alleles per locus in all three species, but never more than two alleles in a single individual. The reads containing polymorphic tetranucleotides were deposited in GenBank under accession numbers KU182396-KU182429.

The number of observed alleles (NA) and observed heterozygosities $(\mathrm{Ho})$ were calculated using Arlequin 3.5 (Excoffier and Lischer, 2010). The probability of identity (PI) and PI between siblings (PIsibs) was calculated using GenAlEx 6.5 (Peakall and Smouse, 2012). PI values estimate the probability that two individuals, taken at random from a population, will have the same genotype at multiple loci (Waits et al., 2001). PI is calculated to determine the number of loci required to resolve individual identity within populations by gradually increasing the number of loci, adding the most variable loci first (Waits et al., 2001). PIsibs is a similar but more conservative estimate of the number of loci required to establish individual identity.

Fifteen polymorphic loci were then evaluated for one population of P. papua $(\mathrm{n}=30)$ and one of $P$. antarcticus $(\mathrm{n}$ $=30$ ). These loci were selected based on the criteria of being polymorphic for all three Pygoscelis species (11 of 15 loci selected) or polymorphic for one or both species (4 loci). The number of observed alleles $\left(N_{A}\right)$ and observed $\left(H_{o}\right)$ and expected heterozygosities $\left(H_{e}\right)$ were calculated using Arlequin 3.5 (Excoffier and Lischer, 2010). Deviations from Hardy-Weinberg equilibrium were calculated with the $F_{I S}$ index in Genetix v. 4.05 (Belkhir et al., 2004).

\section{Results}

For the Pygoscelis genome, a total of 59,889,052 filtered reads and 52,997,551 aligned reads were obtained for $P$. adeliae, 186,606,554 filtered reads and 158,565,602 aligned reads for $P$. papua and 94,746,776 filtered and 80,496,366 aligned reads for $P$. antarcticus (Table 1).

\section{Identification of microsatellite markers}

We identified a total of $35,265,42,057$, and 33,677 microsatellite loci in the genomes of $P$. antarcticus, $P$. papua, and $P$. adeliae, respectively. Among these markers were $17,777,29,618$, and 13,492, respectively, which showed flanking region sequences suitable for primer design. We identified a total of 38,761 dinucleotide, 14,897 trinucleotide and 5,580 tetranucleotide repeats with the
Table 1 - Number of reads (filtered and aligned), number of microsatellite loci and motifs for all three Pygoscelis species, including the total of loci and only those that showed flanking region sequence for primer design.

\begin{tabular}{lccc}
\hline & P.papua & P. antarcticus & P. adeliae \\
\hline Filtered reads & $186,606,554$ & $94,746,776$ & $59,889,052$ \\
Aligned reads & $158,565,602$ & $80,496,366$ & $52,997,551$ \\
No of loci & 42,057 & 35,265 & 33,677 \\
No of loci with primers & 29,618 & 17,777 & 13,492 \\
Total of Dinucleotide & 24,528 & 21,250 & 20,361 \\
Total of Trinucleotide & 11,667 & 9216 & 8,607 \\
Total of Tetranucleotide & 4,370 & 3,788 & 3,691 \\
$\begin{array}{l}\text { Dinucleotide } \\
\text { with primers }\end{array}$ & 18,639 & 11,449 & 8,673 \\
$\begin{array}{l}\text { Trinucleotide } \\
\text { with primers }\end{array}$ & 7,385 & 4,314 & 3,198 \\
$\begin{array}{l}\text { Tetranucleotide } \\
\text { with primers }\end{array}$ & 2,699 & 1,594 & 1,287 \\
\hline
\end{tabular}

primer design in all three genomes (Table 1). The microsatellite loci turned out to be genome-wide distributed across different scaffold (Table S1). Loci motifs, primer sequences, melting temperatures, product size and scaffold for 5,580 tetranucleotides that occur in all three Pygoscelis genomes are included in Table $\mathrm{S} 1$ as well. From these 5,580 tetranucleotide loci, 70 were confirmed to be orthologous for all three species, showing $100 \%$ identity for both primers sequences (forward and reverse) for the same locus and showing the same scaffold and same locus motif (Table S1). However, this number may be underestimated since the comparison considered $100 \%$ identity for both primers designed independently between species. In Pairwise species comparisons we found orthology in 477, 245 and 142 loci between $\mathrm{GP} / \mathrm{CP}, \mathrm{GP} / \mathrm{AP}, \mathrm{AP} / \mathrm{CP}$ respectively.

All species showed higher and similar numbers of dinucleotides, followed by trinucleotides and tetranucleotides (Figure 1). The frequencies of specific repeat motifs were similar for the three species. For dinucleotides, CA repeats were the most frequent across all species, followed by TC, AT and a reduced number of GC loci (Figure $2 \mathrm{~A})$. For trinucleotides, AAT was the most abundant, followed by CCA and GGA (Figure 2B). For tetranucleotides, the most frequent repeat was AAAC, followed by AAAT and AAGG (Figure 2C).

\section{Polymorphism of microsatellite loci for Pygoscelis species}

From the 60,877 primers designed, 170 were selected to evaluate polymorphisms in Pygoscelis: 90 loci based on the $P$. adeliae genome, 40 on $P$. papua and 40 on $P$. antarcticus. Of these, 34 were easily amplified in all three species using the same PCR conditions and were visibly polymorphic at electrophoretic mobility. These 34 loci were also genome wide distributed, being mostly identified in different scaffolds, with exception of six loci which were 


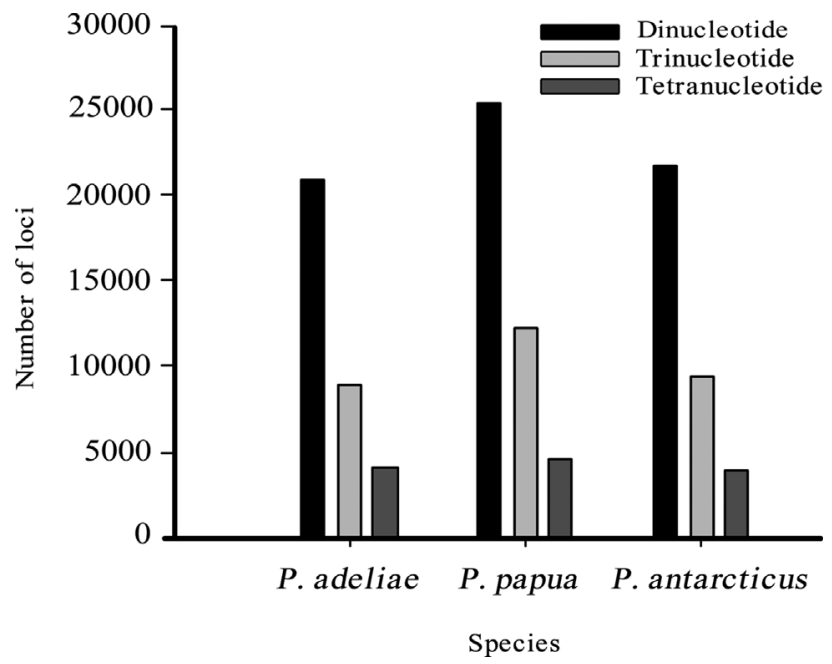

Figure 1 - Numbers of identified dinucleotide (black), trinucleotide (light gray) and tetranucleotide (dark gray) microsatellite repeat loci, for $P$. adeliae, $P$. papua and $P$. antarcticus genome.

found in three different scaffolds (Table 2). Among these 34 loci, 14 were isolated from $P$. adeliae genome, 11 from P. papua, and 9 from $P$. antarcticus. We evaluated these loci in 26 individuals, where 15 loci were polymorphic in all three species, 13 were polymorphic in two species and 6 were polymorphic in one of the three species (Table 2). Therefore, a total of 30 polymorphic microsatellites were identified in $P$. adeliae, 25 in P. papua, and 22 in $P$. antarcticus. Out of all 15 polymorphic loci, the number of alleles per species and per locus varied between 2 and 8 . The highest number of alleles per locus was found in $P$. adeliae (mean 4.87; SD 1.36), followed by P. antarcticus (mean 4.47; SD 1.68) and P. papua (mean 3.53; SD 1.25). Heterozygosity was always higher for $P$. adeliae $\left(H_{o}=\right.$ $0.59)$, followed by $P$. papua $\left(H_{o}=0.54\right)$ and $P$. antarcticus $\left(H_{o}=0.42\right)$ in all 15 loci. Moreover, when we compared the statistics between microsatellite markers isolated from each species' own genome with those isolated from other species' genomes, we found similar ranges of allele numbers and heterozygosity (Table 3 ). This suggests that these markers are not subject to decreased heterozygosity among species of this genus due to cross-species amplification. These results receive support from the orthology of those markers for all three species. Fifty two percent of the microsatellite loci from those 34 selected according to their polymorphism were identified in two or all three Pygoscelis species and $100 \%$ were identified at the same scaffold, and frequently showed the same primer sequence between species. These orthologous percentages were significantly reduced to 3 of 34 loci when we considered $100 \%$ identity for both primers simultaneously.

When evaluating 12 loci for one population of $P$. papua and $P$. antarcticus $(\mathrm{n}=28-30)$, we observed that the number of alleles per species per locus varied between 1 to 7 and 1 to 10 , respectively (Table 4). Only two loci showed
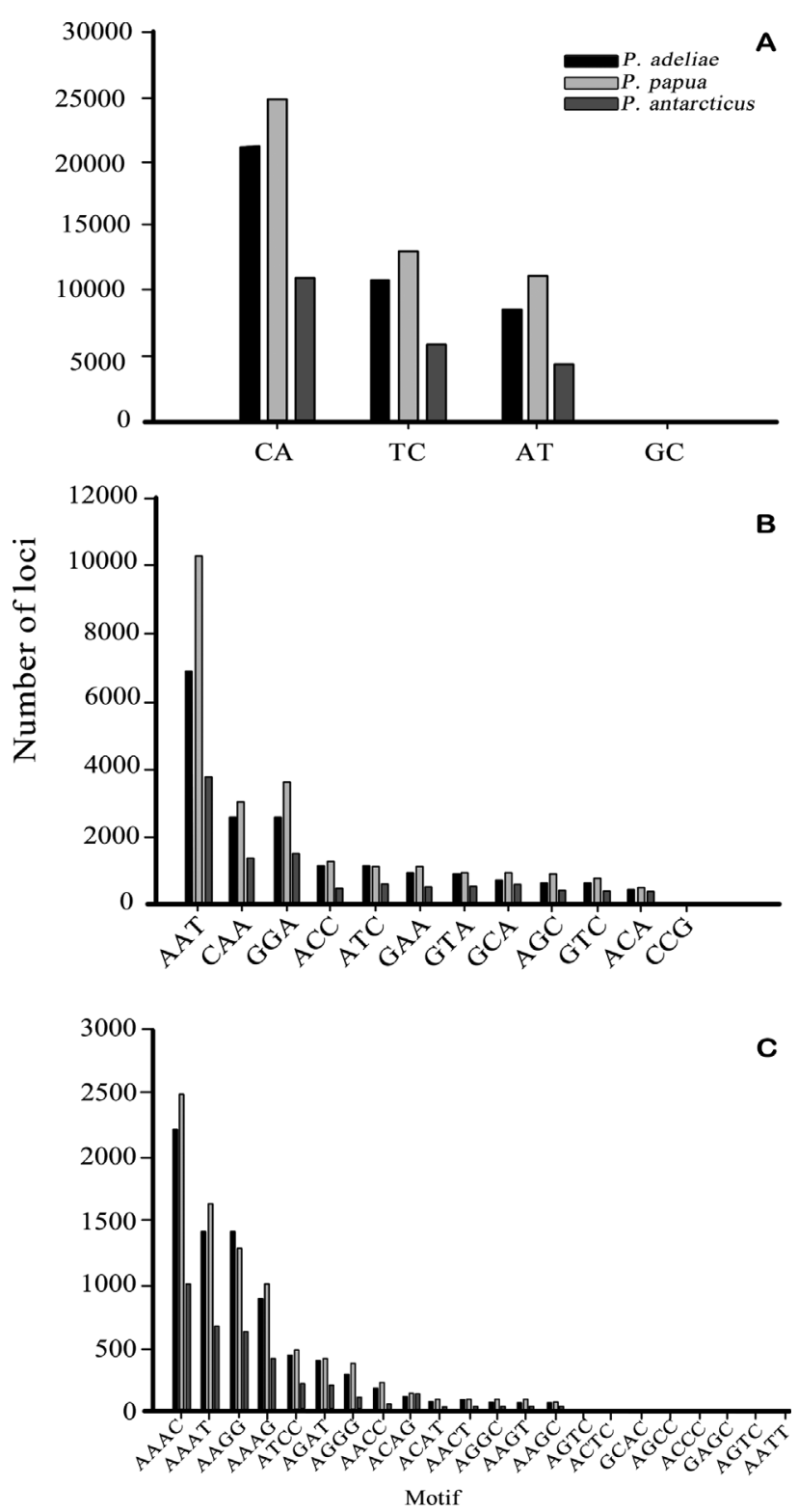

Figure 2 - Number of dinucleotide (A), trinucleotide (B) and tetranucleotide (C) loci with different motifs identified in P. Adeliae (black bar), P. papua (light gray bar) and $P$. antarcticus (dark gray bar).

departure from Hardy-Weinberg Equilibrium for P. papua and three for P. antarcticus. The probability of identity (PI, the probability of two independent samples having an identical genotype), based on all 15 microsatellites, resulted in values as low as $1.4 \mathrm{E}-13,2.5 \mathrm{E}-9$, and 1.2E-11, and PIsibs (probability of identity when random siblings are included in the samples) as low as 5.5E-06, 1.5E-04, and 2.8E-05 for $P$. adeliae, $P$. papua and $P$. antarcticus, respectively (Figure S1). PI was close to zero when combining only the three most polymorphic loci and the same effect was seen in PIsibs when combining the six most polymorphic loci. Markers with the lowest PI are GP-15 (4.8E-02), AP-26 (5.2E-02), and GP-04 (6.7E-02) in P. adeliae; AP-26 


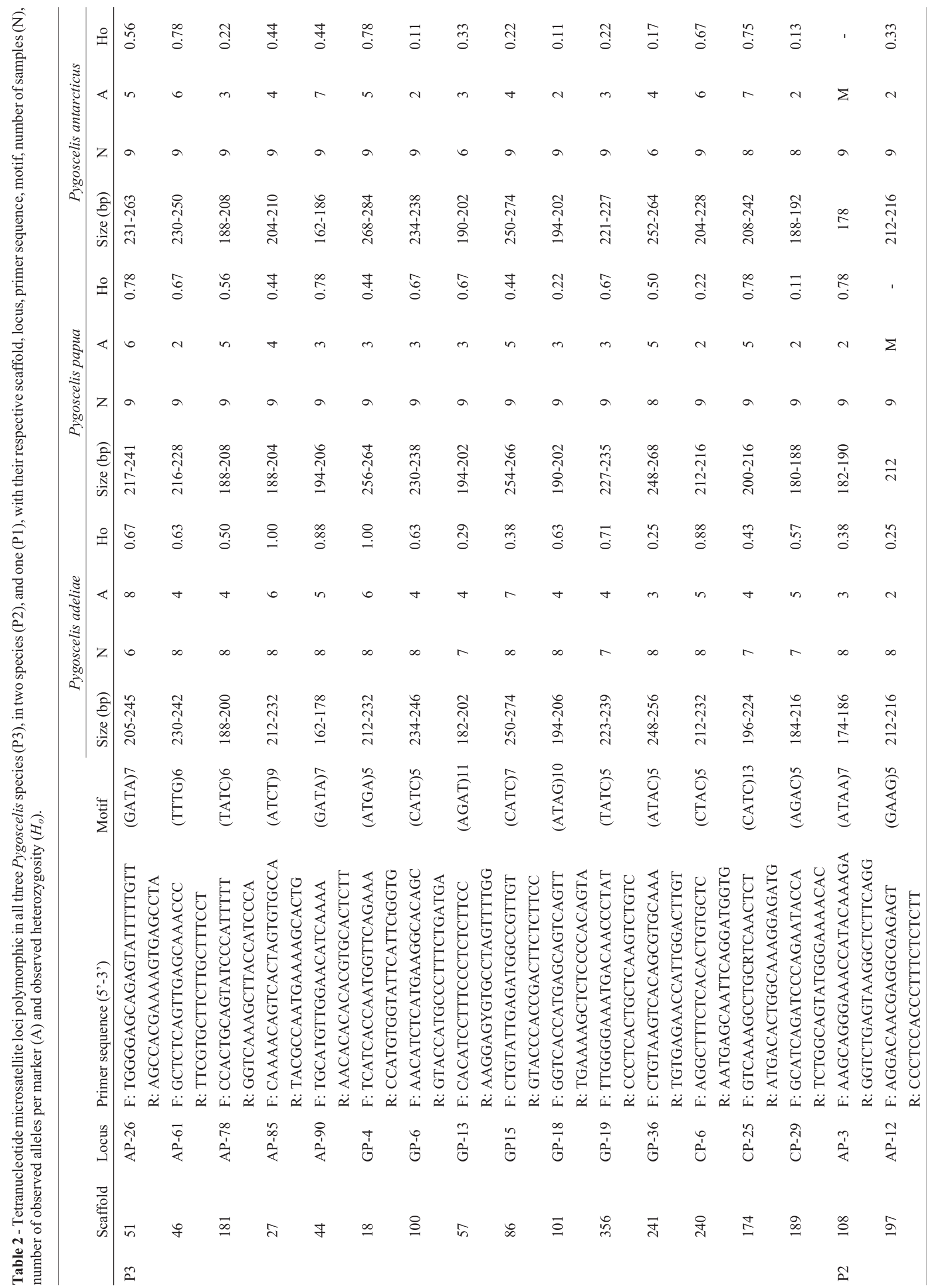




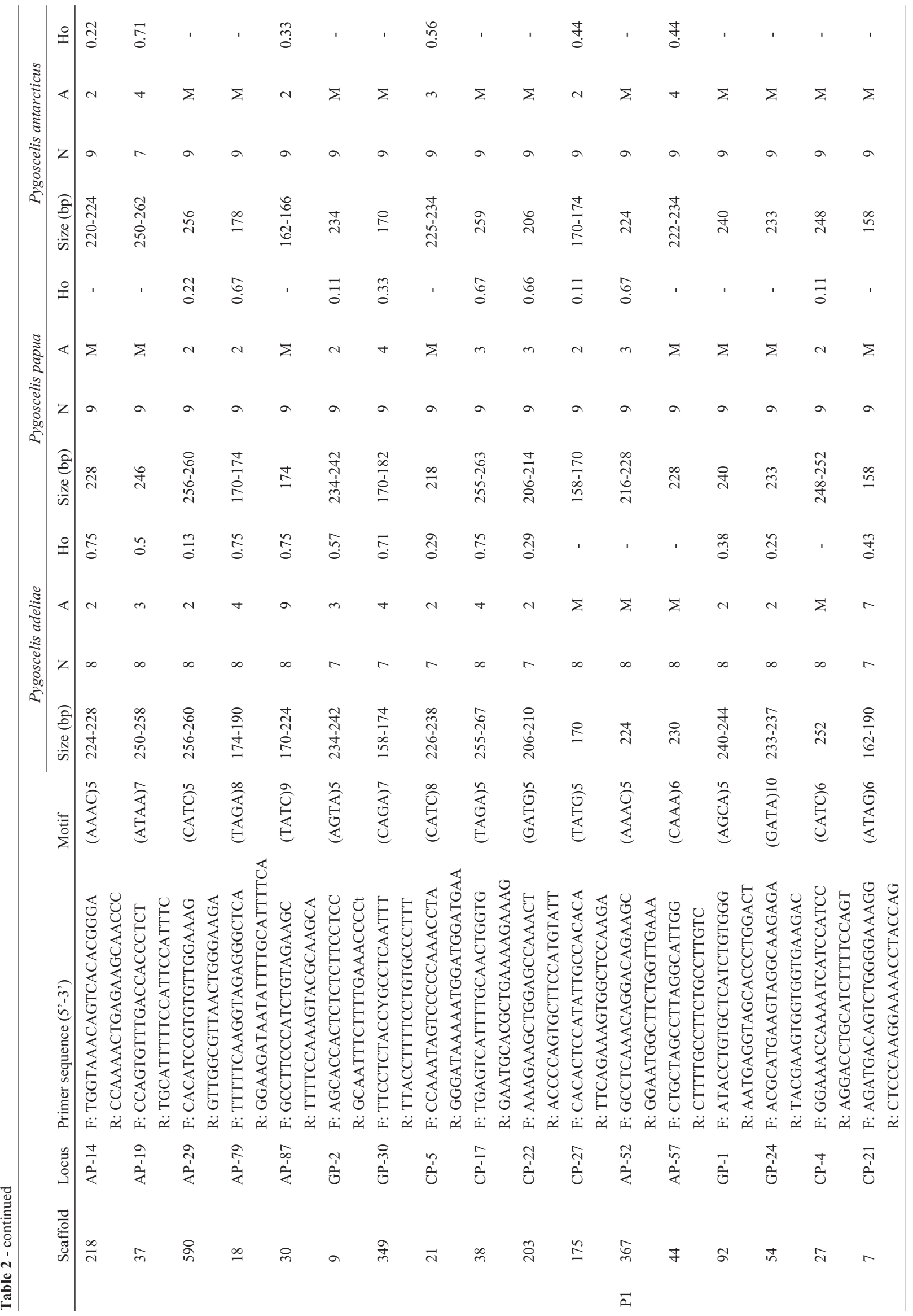


(1.3E-01), CP-25 (1.3E-01), and GP-36 (1.3E-01) in $P . p a-$ pua; and CP-25 (6.9E-02), GP-6 (7.6E-02), and AP-90 (8.4E-02) in $P$. antarcticus.

All microsatellite loci were amplified under the same PCR condition, which means they can be used in multiplex PCR assays. A multiplex microsatellite genotyping run can genotype a total of six loci for each species using three different stains, or eight loci using four stains (e.g. VIC, NED, 6-FAM, PET, HEX, depending on the equipment). We can select loci to be used in all three species according to size range: fragments smaller than 214 bp (AP-78, AP-90, GP13, GP-18) can be combined with fragments greater than 221 bp (GP-6, GP-15, GP-19, GP-36). The remaining loci can be selected for each species individually. Taking $P$. papua as an example, it is possible to combine loci greater than $212 \mathrm{bp}$ in length (CP-06, AP-61, AP-26 and GP-4) with some under 204 bp (AP-85, CP-29).

\section{Discussion}

\section{Comparative analysis of microsatellites in the three genomes}

The SOLiD ${ }^{\mathrm{TM}}$ next-generation sequencing (NGS) platform allowed identification of 110,999 microsatellite loci (di-, tri-, tetranucleotide), with an average of 36,999 loci per Pygoscelis species. Most studies rely on different NGS platforms for screening small parts of the genome for microsatellites. Illumina and 454 platforms have dominated the identification of microsatellite markers in nonmodel organisms (Moodley et al., 2015, Zalapa et al., 2012). Other platforms, such as Ion Torrent PGM and
Table 3 - Mean number of alleles per marker $\left(N_{A}\right)$ and observed heterozygosity $\left(H_{o}\right)$ for microsatellite loci isolated from all three species. (AP: $P$. adeliae, GP: P. papua and CP: P. antarcticus).

\begin{tabular}{|c|c|c|c|c|c|c|}
\hline \multirow{2}{*}{$\begin{array}{l}\text { Microsatellite } \\
\text { loci }\end{array}$} & \multicolumn{2}{|c|}{ P. adeliae } & \multicolumn{2}{|c|}{ P. papua } & \multicolumn{2}{|c|}{ P. antarcticus } \\
\hline & $\mathrm{N}_{\mathrm{A}}$ & $\mathrm{H}_{\mathrm{O}}$ & $\mathrm{N}_{\mathrm{A}}$ & $\mathrm{H}_{\mathrm{O}}$ & $\mathrm{N}_{\mathrm{A}}$ & $\mathrm{H}_{\mathrm{O}}$ \\
\hline $\mathrm{AP}$ & 3.85 & 0.52 & 2.43 & 0.33 & 3.07 & 0.9 \\
\hline GP & 3.91 & 0.53 & 2.91 & 0.40 & 2.82 & 0.25 \\
\hline $\mathrm{CP}$ & 3.56 & 0.23 & 2.33 & 0.22 & 2.67 & 0.17 \\
\hline
\end{tabular}

Illumina MiSeq, or the single-molecule real-time DNA sequencing platform PacBio (Pacific Biosciences), have recently been gaining space as a means of rapid, small scale microsatellite development (Wei et al., 2014).

We used the SOLiD platform to sequence three penguin genomes and not only search for microsatellite loci, but also characterize them according to polymorphism, distribution in the genome and orthology between species, with the aim of contributing to the increase in the number of comparative studies between Pygoscelis species using markers with high resolution.

We observed that the number of repeats and motifs varied between the three Pygoscelis species. We found large amounts of dinucleotides, followed by tri- and tetranucleotides in Pygoscelis, as described in other vertebrate genomes (e.g., Huang et al., 2015). However, the relative abundance of different motif classes (e.g. dinucleotide) and motif types (e.g. AT, ACC, AAAT) is not conserved when compared with other vertebrate species. For example, Castoe et al. (2010) identified in the copperhead snake (Agkistrodon contortrix) a total of 14,612 simple sequence

Table 4 - Tetranucleotide microsatellite loci polymorphism for 30 individuals and 12 loci for $P$. papua and $P$. antarcticus, (N: Number of observed alleles per marker; $H_{o}$ Observed heterozygosity, $H_{e}$ : Expected heterozygosity, $F_{I S}$ value). ${ }^{*}$ Significant $F_{I S}$ values, $\mathrm{p}<0.05$.

\begin{tabular}{|c|c|c|c|c|c|c|c|c|c|c|c|c|}
\hline \multirow[b]{2}{*}{ Locus } & \multicolumn{6}{|c|}{ Pygoscelis papua } & \multicolumn{6}{|c|}{ Pygoscelis antarcticus } \\
\hline & Size (bp) & $\mathrm{N}$ & $\mathrm{A}$ & Ho & $\mathrm{He}$ & $F_{\text {IS }}$ & Size (bp) & $\mathrm{N}$ & $\mathrm{A}$ & Но & $\mathrm{He}$ & $F_{\mathrm{IS}}$ \\
\hline AP-3 & $178-186$ & 30 & 2 & 0.37 & 0.49 & $0.261 *$ & $174-178$ & 30 & 2 & 0.067 & 0.065 & -0.018 \\
\hline AP-19 & 246 & 30 & 1 & 0 & 0 & & $250-290$ & 28 & 8 & 0.82 & 0.79 & -0.034 \\
\hline AP-26 & $225-241$ & 30 & 6 & 0.70 & 0.77 & 0.100 & $231-255$ & 30 & 6 & 0.8 & 0.8 & 0 \\
\hline AP-61 & & & & & & & $218-250$ & 28 & 8 & 0.643 & 0.755 & $0.151^{*}$ \\
\hline AP-78 & $188-212$ & 30 & 7 & 0.80 & 0.69 & -0.169 & $188-208$ & 30 & 4 & 0.266 & 0.38 & $0.309 *$ \\
\hline AP-85 & $188-208$ & 30 & 5 & 0.67 & 0.70 & 0.051 & $200-224$ & 30 & 7 & 0.700 & 0.763 & 0.084 \\
\hline AP-90 & $194-214$ & 30 & 6 & 0.53 & 0.56 & 0.045 & $158-190$ & 30 & 9 & 0.900 & 0.840 & -0.072 \\
\hline GP-6 & $230-234$ & 29 & 2 & 0.28 & 0.29 & 0.051 & 234 & 28 & 1 & 0 & 0 & \\
\hline GP-13 & $190-206$ & 30 & 5 & 0.90 & 0.62 & -0.479 & & & & & & \\
\hline GP15 & & & & & & & $250-286$ & 30 & 10 & 0.767 & 0.806 & 0.049 \\
\hline GP-36 & $240-260$ & 30 & 5 & 0.13 & 0.16 & $0.171 *$ & $244-260$ & 30 & 5 & 0.333 & 0.473 & $0.30 *$ \\
\hline CP-6 & & & & & & & $228-236$ & 30 & 3 & 0.600 & 0.524 & -0.146 \\
\hline CP-17 & $255-259$ & 29 & 2 & 0.10 & 0.09 & -0.037 & & & & & & \\
\hline CP-22 & $190-234$ & 30 & 7 & 0.73 & 0.76 & 0.311 & & & & & & \\
\hline CP-25 & $192-212$ & 30 & 6 & 0.63 & 0.55 & -0.152 & $204-268$ & 30 & 10 & 0.866 & 0.826 & -0.05 \\
\hline
\end{tabular}


repeats (SSRs), 4,564 of which had flanking sequences suitable for 454 shotgun genome sequencing. These represent various amounts of different motif classes, with numbers of tetranucleotides, followed by trinucleotides and dinucleotides. The most frequent motifs were also different from those we found in our study (TC, ATT, ATCT; Castoe et al., 2010). McCulloch and Stevens (2011) used Roche FLX (Titanium) Genome Sequencing to identify microsatellites (29,721 di-, tri-, tetranucleotide) for phyllostomid bats, and the authors found a majority of tetranucleotides, followed by dinucleotides and trinucleotides. Recently, 48 bird species genomes, representing all orders of Aves, were completed for phylogenetic reconstruction, which also provide a large amount of data and microsatellites to be evaluated for comparative analyses (Jarvis et al., 2014).

Primers for tetranucleotides in Pygoscelis were designed with the same annealing temperature to enable multiplex PCR assays, which is a more affordable and timeefficient technique to genotype multiple loci and individuals. In this way, it is possible to genotype up to eight loci for each individual in a single run. Moreover, the small size $(<$ $200 \mathrm{bp}$ ) of several fragments identified in the Pygoscelis genome allows easy amplification of low quality or low concentration DNA, such as that extracted from non-invasive (e.g. feather, feces) or ancient (e.g. museum or palaentological) samples. To this date, non-invasive DNA samples have not been used to conduct population genetics or ecological studies in penguin species due to the difficulty of obtaining good quality material for nuclear marker amplification. These samples have mostly been used for studying penguin diets, sex identification (e.g., Jarman et al., 2002; Deagle et al., 2007; Constantini et al., 2008), or studying the evolution of species using mtDNA from sub-fossil bones (e.g. Lambert et al., 2002; Ritchie et al., 2004).

In this study, we provide 34 novel polymorphic microsatellite markers, 15 of which are polymorphic in all three species and can easily be genotyped using multiplex PCR. Recently, Moodley et al. (2015) used NGS to develop markers for the thin-billed prion (Pachyptila belcheri), a sub-Antarctic seabird, and evaluated cross-amplification in six other seabird species, finding that heterozygosity decreased while proportion of non-amplifying loci increased with phylogenetic distance. In our case, similar heterozygosity was observed for all Pygoscelis species using loci cross-amplification, which suggests that these markers are not subject to the heterozygosity decrease among closely related species. Moreover, this was confirmed by the orthology of most loci selected for all species with same or similar primer sequences.

These polymorphic markers allow individual discrimination and their suitability for use in non-invasive or museum samples promotes their application in different types of research, such as species monitoring in the face of climate change, parentage analyses, determination of sample origin and of population genetics structure. Moreover, the same markers can be employed in comparative phylogeographical and population genetics studies of Pygoscelis species, given that the 15 loci developed in this study easily cross-amplify, are orthologous and widely distributed in the genome.

\section{Polymorphisms and utility for investigation of penguin populations}

We report a large number of microsatellite loci identified in the genomes of three Pygoscelis species using NGS and classified according to their level of polymorphism, which may also be applied to the study of other penguin species. Previously, only a limited number of microsatellite loci were available for penguins. Twelve loci had been developed for the Humboldt penguin (Schlosser et al., 2003; 2009), five for Spheniscus species (Akst et al., 2002), and six for the Adélie penguin (Roeder et al., 2001; 2002). These had been identified using traditional methods and possessed varying degrees of polymorphism in different species. In general, the same markers from these three studies have been used in population genetics investigations of several different penguin species.

Although penguins have been associated with philopatric behavior, which is often correlated with significant population structure, all studies so far have shown little or no population structure. Absence of population genetic structure was observed in Galapagos penguins using five microsatellite loci (Nims et al., 2008), while Bouzat et al. (2009), using only four loci in samples from six colonies of Magellanic penguins, found limited genetic structure. Roeder et al. (2001) analyzed seven microsatellite loci in Adélie penguin samples collected from 13 localities and did not identify genetic differentiation between colonies around the Antarctic continent, with pairwise $F_{s t}$ below 0.02 and an overall $F_{s t}$ equal to 0.0007 . Trakiiska et al. (2005) evaluated three loci in P. papua from Livingston Island, however, only one of them was polymorphic. Overeem et al. (2008) studied the population genetics of Eudyptula minor using five loci in samples from seven colonies and found that most were genetically homogeneous. Likewise, Schlosser et al. (2009) found reduced genetic structure in the Humboldt penguin along its distribution based on 12 microsatellite loci. Sakaoka et al. (2014) used eight microsatellite loci to understand the breeding behavior of $P$. adeliae, revealing an absence of extra-pair paternity (EPP) in a particular captive population. All the aforementioned studies used an average of 6 loci for penguin population genetics studies, most of which using the same or similar marker combination. If these markers do not provide enough resolution to reflect species behavior and ecology, then doubt is cast on the studies' findings. Recently, Vianna et al. (2017), used a total of 12 loci selected from this study (Table 4) to understand population genetics structure of $P$. papua. Significant population structure was identified between $P$. papua colonies from sub-Antarctic 
(Falkland/Malvinas, Crozet and Kerguelen Islands) and those from WAP, but also among nine localities along WAP. These results put in evidence the resolution of our selected markers to detect population genetics structure in this species. Other loci developed for penguins were often not evaluated in a population study or showed population structure. Ahmed et al. (2009) developed 25 loci for the Macaroni penguin, eight to 12 loci were found to be polymorphic, and Kang et al. (2015) isolated 16 polymorphic microsatellite loci for the Chinstrap penguin $(P$. antarcticus). However, those markers were not employed in population genetics investigations. Boessenkool et al. (2008, 2009) developed 12 other microsatellite loci for the Yellow-eyed penguin (Megadyptes antipodes), and found two distinct genetic groups based on that dataset.

This elicits different questions: are penguins really philopatric, highly migrant, or both? Are species with such large geographical distributions, living under different environmental conditions and subject to various barriers, really that genetically homogeneous? Or should more highly variable markers be used to obtain the appropriate resolution for the type of questions asked? For several kinds of population genetics data analyses, results are sensitive to the number of loci used and their polymorphic information content, as well as the number of populations sampled and of individuals typed in each population. Evanno et al. (2005), for instance, suggest that a minimum of 10 polymorphic microsatellites are required to detect population genetic structure using the Bayesian algorithm implemented in Structure software.

There is an increasing amount of research using SNPs with genomic methods, such as GBS, RADseq or ddRAD for population studies, including recent studies in penguins (e.g. Clucas et al., 2016; Cristofari et al., 2016). However, microsatellites will remain useful for population studies when discrepant patterns are observed between markers (mtDNA, microsatellite, SNPs). This is the case of three clades identified for the emperor penguin using mtDNA (Younger et al., 2015) and the lack of population structure using genome-wide SNPs (Cristofari et al., 2016). Apart of population studies, microsatellites are very useful for studies such as individual-based identification or paternity assessment. Therefore, it is important to identify polymorphic microsatellite loci in non-model species, especially those inhabiting areas highly impacted by climate change, such as the Antarctic. We identified about 11,849 tetranucleotide markers and selected 34 which were polymorphic, cross-amplified in all three Pygoscelis species, and did not show signs of heterozygosity reduction due to cross-amplification or due to orthology identified in the majority of those loci selected. We believe that the microsatellite markers developed in this study will help to gain a comprehensive understanding of Pygoscelis penguin population genetics and ecology.

\section{Acknowledgement}

We acknowledge the financial support of Fondecyt (No 11110060 and 1150517), the Chilean Antarctic Institute (INACH T_12-13, G_06-11, RT_12-14) and CNPq (482501/2013-8).

\section{References}

Ahmed S, Hart T, Dawson DA, Horsburgh GJ, Trathan PN and Rogers AD (2009) Isolation and characterization of macaroni penguin (Eudyptes chrysolophus) microsatellite loci and their utility in other penguin species (Spheniscidae, AVES). Mol Ecol Resour 9:1530-1535.

Akst EP, Boersma PD and Fleischer RC (2002) A comparison of genetic diversity between Galápagos Penguin and the Magellanic Penguin. Conserv Genet 3:375-383.

Aljanabi SM and Martinez I (1997) Universal and rapid saltextraction of high quality genomic DNA for PCR-based techniques. Nucleic Acids Res 25:4692-4693.

Boessenkool S, King TM, Seddon PJ and Waters JM (2008) Isolation and characterization of microsatellite loci from the yellow-eyed penguin (Megadyptes antipodes). Mol Ecol Resour 8:1043-1045.

Boessenkool S, Star B, Waters JM and Seddon PJ (2009) Multilocus assignment analyses reveal multiple units and rare migration events in the recently expanded yellow-eyed penguin (Megadyptes antipodes). Mol Ecol 18:2390-2400.

Bouzat JL, Walker BG and Boersma PD (2009) Regional genetic structure in the Magellanic penguin (Spheniscus magellanicus) suggests metapopulation dynamics. Auk 126:326-334.

Brody JR and Kern SE (2004) Sodium boric acid: A Tris-free, cooler conductive medium for DNA electrophoresis. BioTechniques 36:214-216.

Castoe TA, Poole AW, Gu W, Jason de Koning AP, Daza JM, Smith EN and Pollock DD (2010) Rapid identification of thousands of copperhead snake (Agkistrodon contortrix) microsatellite loci from modest amounts of 454 shotgun genome sequence. Mol Ecol Resour 10:341-347.

Clucas GV, Dunn MJ, Dyke GJ, Emslie SD, Naveen R, Polito MJ, Pybus OG, Rogers AD and Hart T (2014) A reversal of fortunes: Climate change 'winners' and 'losers' in Antarctic Peninsula penguins. Sci Rep 4:5024.

Clucas GV, Younger JL, Kao D, Rogers AD, Handley J, Miller GD, Jouventin P, Nolan P, Gharbi K, Miller KJ, et al. (2016) Dispersal in the sub-Antarctic: King penguins show remarkably little population genetic differentiation across their range. BMC Evol Biol 16:211.

Constantini V, Guaricci AC, Laricchiuta P, Rausa F and Lacalandra GM (2008) DNA sexing in Humboldt penguins (Spheniscus humboldti) from feather samples. Anim Reprod Sci 106:162-167.

Cristofari R, Bertorelle G, Ancel A, Benazzo A, Le Maho Y, Ponganis PJ, Stenseth NC, Trathan, PN, Whittington JD, Zanetti E, et al. (2016) Full circumpolar migration ensures evolutionary unity in the Emperor penguin. Nat Commun $7: 11842$.

Croxall JP, Trathan PN and Murphy EJ (2002) Environmental change and Antarctic seabird populations. Science 297:1510-1514. 
Dakin EE and Avise JC (2004) Microsatellite null allele in parentage analysis. Heredity 93:504-509.

Dawson DA, Horsburgh GJ, Küpper C, Stewart IRK, Ball AD, Durrant KL, Hansson B, Bacon I, Bird S, Klein A, et al. (2010) New methods to identify conserved microsatellite loci and develop primer sets of high cross-species utility - As demonstrated for birds. Mol Ecol Resour 10:475-494.

Deagle BE, Gales NJ, Evans K, Jarman SN, Robinson S, Treblico R and Hindell MA (2007) Studying seabird diet through genetic analysis of faeces: A case study on Macaroni penguins (Eudyptes chrysolophus). PLoS One 2:e831.

Dewoody J, Nason JD and Hipkins VD (2006) Mitigating scoring errors in microsatellite data from wild populations. Mol Ecol Notes 6:951-957.

Ellegren H (2008) Sequencing goes 454 and takes large-scale genomics into the wild. Mol Ecol 17:1629-1631.

Evanno G, Regnaut S and Goudet J (2005) Detecting the number of clusters of individuals using the software structure: A simulation study. Mol Ecol 14:2611-2620.

Excoffier L and Lischer HE (2010) Arlequin suite version 3.5: A new series of programs to perform population genetics analyses under Linux and Windows. Mol Ecol Resour 10:564567.

Freer JJ, Mable BK, Clucas G, Rogers AD, Polito MJ, Dunn M, Naveen R, Levy H and Hart T (2015) Limited genetic differentiation among Chinstrap penguin (Pygoscelis antarctica) colonies in the Scotia Arc and Western Antarctic Peninsula. Polar Biol 38:1493-1502.

Greig C, Jacobson DP and Banks MA (2003) New tetranucleotide microsatellites for fine-scale discrimination among endangered Chinook salmon (Onchorhynchus tshawytscha). Mol Ecol Notes 3:376-379.

Huang J, Li Y-Z and Du L-M (2015) Genome-wide survey and analysis of microsatellites in Giant Panda (Ailuropoda melanoleuca), with a focus on the applications of a novel microsatellite marker system. BMC Genomics 16:61.

Jarman SN, Gales NJ, Tierney M, Gill PC and Elliott NG (2002) A DNA-based method for identification of krill species and its application to analyzing the diet of marine vertebrate predators. Mol Ecol 11:2679-2690.

Jarvis ED, Siavash M, Aberer AJ, Li B, Houde P, Li C, Ho SYW, Faircloth BC, Nabholz B, Howard JT, et al. (2014) Whole genome analyses resolve the early branches in the tree of life of modern birds. Science 346:1320-1331.

Kang S, Jung J-W, Ahn DH, Lee JE, Kim J-H and Park H (2015) Isolation and characterization of polymorphic microsatellite loci from the chinstrap penguin, Pygoscelis antarctica. Conserv Genet Resour 7:561-563.

Kruglyak S, Durrett RT, Schug MD and Aquadro CF (1998) Equilibrium distributions of microsatellite repeat length resulting from a balance between slippage events and point mutations. Proc Natl Acad Sci U S A 95:10774-10778.

Lambert DM, Ritchie PA, Millar CD, Holland B, Drummond AJ and Baroni C (2002) Rates of evolution in ancient DNA from Adelie penguins. Science 295:2270-2273.

Li C, Zhang Y, Li J, Kong L, Kong L, Hu H, Pan H, Xu L, Deng Y, Li Q, et al. (2014) Two Antarctic penguin genomes reveal insights into their evolutionary history and molecular changes related to the Antarctic environment. GigaScience 3:27.

Li H, Handsaker B, Wysoker A, Fennell T, Ruan J, Homer N, Marth G, Abecasis G and Durbin R (2009) The Sequence
Alignment/Map format and SAM tools. Bioinformatics 25:2078-2079.

McCulloch ES and Stevens RD (2011) Rapid development and screening of microsatellite loci for Artibeus lituratus and their utility for six related species within Phyllostomidae. Mol Ecol Resour 11:903-913.

Meredith MP and King JC (2005) Rapid climate change in the ocean west of the Antarctic Peninsula during the second half of the 20th century. Geophys Res Lett 32:1-5.

Montes-Hugo M, Doney SC, Ducklow HW, Fraser W, Martinson D, Stammerjohn SE and Schofield O (2009) Recent changes in phytoplankton communities associated with rapid regional climate change along the Western Antarctic Peninsula. Science 323:1470-1473.

Moodley Y, Masello JF, Cole TL, Calderon L, Munimanda GK, Thali MR, Alderman R, Cuthbert RJ, Marin M, Massaro M, et al. (2015) Evolutionary factors affecting the cross-species utility of newly developed microsatellite markers in seabirds. Mol Ecol Resour 15:1046-1058.

Neff BD and Gross MR (2001) Microsatellite evolution in vertebrates: Inference from AC dinucleotide repeats. Evolution 55:1717-1733.

Nims BD, Vargas FH, Merkel J and Parker PG (2008) Low genetic diversity and lack of population structure in the endangered Galápagos penguin (Spheniscus mendiculus). Conserv Genet 9:1413-1420.

Overeem RL, Peucker AJ, Austin CM, Dann P and Burridge CP (2008) Contrasting genetic structuring between colonies of the World's smallest penguin, Eudyptula minor (Aves: Spheniscidae). Conserv Genet 9:893-905.

Peakall R and Smouse PE (2012) GenAlEx 6.5: Genetic analysis in Excel. Population genetic software for teaching and research - An update. Bioinformatics 28:2537-2539.

Peña FM, Poulin E, Dantas GPM, González-Acuña D, Petry MV and Vianna JA (2014) Have historical climate changes affected Gentoo penguin (Pygoscelis papua) populations in Antarctica? PLoS One 9:e95375.

Primmer CR, Raudsepp T, Chowdhary BP, Møller AP and Ellegren $\mathrm{H}$ (1997) A low frequency of microsatellite in the avian genome. Genome Res 7:471-482.

Ritchie PA and Lambert DM (2000) A repeat complex in the mitochondrial control region of Adélie penguins from Antarctica. Genome 43:613-618.

Ritchie PA, Millar, CD, Gibb GC, Baroni C and Lambert DM (2004) Ancient DNA enables timing of the Pleistocene origin and Holocene expansion of two Adélie penguin lineages in Antarctica. Mol Biol Evol 21:240-248.

Roeder AD, Marshall RK, Mitchelson AJ, Visagathilagar T, Ritchie PA, Love DR, Pakai TJ, McPartlan HC, Murray ND, Robinson NA, et al. (2001) Gene flow on the ice: Genetic differentiation among Adélie penguin colonies around Antarctica. Mol Ecol 10:1645-1646.

Roeder AD, Ritchie PA and Lambert DM (2002) New DNA markers for penguins. Conserv Genet 3:341-344

Rozen S and Skaletsky H (2000) Primer3 on the WWW for general users and for biologist programmers. Methods Mol Biol 132:365-386.

Sakaoka K, Suzuki I, Kasugai N and Fukumoto Y (2014) Paternity testing using microsatellite DNA markers in captive Adélie penguins (Pygoscelis adeliae). Zoo Biol 33:463-470. 
Schlosser JA, Garner TWJ, Dubach JM and Mcelligott AG (2003) Characterization of microsatellite loci in Humboldt penguin (Spheniscus humboldti) and cross-amplification in other penguin species. Mol Ecol Notes 3:62-64.

Schlosser JA, Dubach JM, Garner TWJ, Araya B, Bernal M, Simeone A, Smith KA and Wallace RS (2009) Evidence for gene flow differs from observed dispersal patterns in the Humboldt penguin, Spheniscus humboldti. Conserv Genet 10:839-849.

Schuelke M (2000) An economic method for the fluorescent labeling of PCR fragments. Nature Biotechnol 18:233-234.

Stonehouse B (1975) Introduction: The Spheniscidae. In: Stonehouse B (ed) The Biology of Penguins. Macmillan Press, London, pp 1-15.

Schug MD, Hutter CM, Wetterstrand KA, Gaudette MS, Mackay TFC and Aquadro CF (1998) The mutation rates of di-, triand tetranucleotide repeats in Drosophila melanogaster. Mol Biol Evol 15:1751-1760.

Trakiiska E, Bichev S, Telegeev G, Dybkov M, Bezrukov V, Jankov J and Savov A (2005) Study of three DNA microsatellite markers in Pygoscelis papua penguins (Aves: Sphenisciformes) from the Livingston Island. Acta Zool Blug 57:333-340.

Trathan PN, Fretwell PT and Stonehouse B (2011) First recorded loss of an Emperor penguin colony in the recent period of Antarctic regional warming: Implications for other colonies. PloS One 6:e14738.

Vianna JA, Noll D, Dantas GPM, Petry MV, Barbosa A, González-Acuña D, Le Bohec C, Bonnadona F and Poulin E (2017) Marked phylogeographic structure of Gentoo penguin reveals an ongoing diversification process along the Southern Ocean. Mol Phylogenet Evol 107:486-498.

Waits LP, Luikart G and Taberlet P (2001) Estimating the probability of identity among genotypes in natural populations: Cautions and guidelines. Mol Ecol 10:249-256.

Younger JL, Clucas GV, Kooyman G, Wienecke B, Rogers AD, Trathan PN, Hart T and Miller KJ (2015) Too much of a good thing: Sea ice extent may have forced emperor penguins into refugia during the last glacial maximum. Global Change Biol 21:2215-2226.

Zalapa JE, Cuevas H, Zhu H, Steffan S, Senalik D, Zeldin E, McCown B, Harbut R and Simon P (2012) Using next-gene- ration sequencing approaches to isolate simple sequence repeat (SSR) loci in the plant sciences. Am J Bot 99:193-208.

Zhang G, Li C, Li Q, Li B, Larkin DM, Lee C, Storz JF, Antunes A, Greenwold MJ, Meredith RW, et al. (2014) Comparative genomics across modern bird species reveal insights into panavian genome evolution and trait biodiversity. Science 346:1311-1320.

Wei N, Bemmels JB and Dick CW (2014) The effects of read length, quality and quantity on microsatellite discovery and primer development:From Illumina to PacBio. Mol Ecol Resour 14:953-965.

Williams TD (1995) The Penguins: Spheniscidae. Oxford University Press, Oxford, 295 p.

\section{Internet Resources}

AmplifX 1.5.4 by Nicolas Jullien; CNRS, Aix-Marseille Université. http://crn2m.univ-mrs.fr/pub/amplifx-dist.

Belkhir K, Borsa P, Chikhi L, Raufaste N and Bonhomme F (2004) Genetix 4.05, logiciel sous Windows pour la genetique despopulations. Laboratoire Genome, Populations, Interactions, CNRSUMR 5000. Université de Montpellier II, Montpellier, France. http://www.genetix.univ-montp2.fr/genetix/genetix.htm.

IUCN - International Union for Conservation of Nature (2017) IUCN Red List of Threatened Species, version 2017. http://www.iucnredlist.org (accessed June 16, 2016).

Li, H. (2012). Seqtk Toolkit for processing sequences in FASTA/Q formats (https://github.com/lh3/seqtk).

\section{Supplementary Material}

The following online material is available for this article: Figure S1 - Probability of identity (PI), and the PI between siblings $\left(\mathrm{PI}_{\mathrm{sib}}\right)$ for the 15 microsatellite loci.

Table S1 - All tetranucleotide loci for $P$. adeliae, $P$. papua P. antarcticus.

Associate Editor: Houtan Noushmehr

License information: This is an open-access article distributed under the terms of the Creative Commons Attribution License (type CC-BY), which permits unrestricted use, distribution and reproduction in any medium, provided the original article is properly cited. 